\title{
Author Index Volume 45 (2015)
}

The issue number is given in front of the pagination

Abbas, M.A.A., see Khedr, E. (1) 117-126

Abdin, E., see Subramaniam, M. (4) 1127-1138

Abe, K., see Matsuzono, K. (3) 771-780

Acedo-Jiménez, C., see Olazarán, J. (4) 1157-1173

Agostinho, P., A. Pliássova, C.R. Oliveira and R.A. Cunha, Localization and Trafficking of Amyloid$\beta$ Protein Precursor and Secretases: Impact on Alzheimer's Disease (2) 329-347

Alegret, M., see Moreno-Grau, S. (2) 621-629

Allouche, A., see Pinçon, A. (1) 195-204

Almkvist, O., see Leuzy, A. (4) 1077-1088

Altamura, C., see Buratti, L. (3) 883-890

Altavilla, R., see Vecchio, F. (3) 745-756

Anderson, S.J., see Koenig, A.M. (1) 175-185

Andrés, M.V., see Lovestone, S. (1) 75-88

Antúnez, C., see Moreno-Grau, S. (2) 621-629

Antúnez, C., see Olazarán, J. (4) 1157-1173

Arahata, Y., see Ito, K. (2) 543-552

Ardekani, B.A., see Elahi, S. (3) 921-931

Armand-Ugón, M., E. Aso, J. Moreno, M. Riera-Codina, A. Sánchez, E. Vegas and I. Ferrer, Memory Improvement in the A $\beta P P / P S 1$ Mouse Model of Familial Alzheimer's Disease Induced by Carbamylated-Erythropoietin is Accompanied by Modulation of Synaptic Genes (2) 407-421

Arnold, S.E., see Koenig, A.M. (1) 175-185

Arroyo-Anlló, E.M., P. Ingrand, J.-P. Neau and R. Gil, Procedural Learning of Semantic Categorization in Parkinson's Disease (1) 205-216

Asada, T., see Bun, S. (1) 15-25

Asada-Utsugi, M., see Jingami, N. (1) 109-115

Aso, E., see Armand-Ugón, M. (2) 407-421

Assini, A.G., see Miranda, L.F.J.R. (2) 609-620

Attar, G.A., see Khedr, E. (1) 117-126

Baches, S., see Richard, B.C. (4) 1223-1236

Bachman, A.H., see Elahi, S. (3) 921-931

Balestrini, S., see Buratti, L. (3) 883-890

Barbera, C., see Leandri, M. (3) 705-707

Barmada, M.M., see Wang, X. (2) 651-658
Barneda, B., see Moreno-Grau, S. (2) 621-629

Bastard, N.L., see Niemantsverdriet, E. (4) 1039-1043

Bastin, C., see Moyse, E. (2) 631-638

Baudron, C.R., L. Chambonnier, A. Buissionnière, A. Giese, N. Macrez, Y. Cho, V. Fénelon, L. Blaszczyk, P. Dubus, P. Lehours, F. Mégraud, N. Salles and C. Varon, An Eighteen-Month Helicobacter Infection Does Not Induce Amyloid Plaques or Neuroinflammation in Brains of Wild Type C57BL/6J Mice (4) 1045-1050

Bayer, T.A., see Meißner, J.N. (2) 471-482

Bayer, T.A., see Richard, B.C. (4) 1223-1236

Bechtel, C., see Remington, R. (2) 395-405

Becker, J.T., see Koenig, A.M. (1) 175-185

Becker, J.T., see Wang, X. (2) 651-658

Begley, A.E., see Koenig, A.M. (1) 175-185

Bennett Jr, J.P., see Rice, A.C. (4) 1051-1059

Benzinger, T., see Hsu, P.J. (1) 27-33

Berg, A.I., see Rolstad, S. (3) 873-881

Berisha, V., S. Wang, A. LaCross and J. Liss, Tracking Discourse Complexity Preceding Alzheimer's Disease Diagnosis: A Case Study Comparing the Press Conferences of Presidents Ronald Reagan and George Herbert Walker Bush (3) 959-963

Bermejo-Pareja, F., see Olazarán, J. (4) 1157-1173

Bernabei, R., see Silveri, M.C. (2) 483-494

Bezprozvanny, I., see Zhang, H. (2) 561-580

Biessels, G.J., see Wisse, L.E.M. (4) 1247-1256

Billinger, S.A., see Watts, A. (1) 187-193

Blanc, F., see Cretin, B. (2) 521-526

Blanchard, J., see Wang, X. (2) 423-435

Blankenstein, M.A., see Jongbloed, W. (1) 35-43

Blaszczyk, L., see Baudron, C.R. (4) 1045-1050

Block, W., see Meiberth, D. (1) 139-146

Blond, E., see Revel, F. (2) 553-560

Boada, M., see Lovestone, S. (1) 75-88

Boada, M., see Moreno-Grau, S. (2) 621-629

Boecker, H., see Meiberth, D. (1) 139-146

Boncoraglio, G.B., F. Piazza, M. Savoiardo, L. Farina, J.C. DiFrancesco, S. Prioni, F. Tagliavini, E.A. 
Parati and G. Giaccone, Prodromal Alzheimer's Disease Presenting as Cerebral Amyloid Angiopathy-Related Inflammation with Spontaneous Amyloid-Related Imaging Abnormalities and High Cerebrospinal Fluid Anti-A $\beta$ Autoantibodies (2) 363-367

Bonnefoi, B., see Moreau, N. (2) 581-597

Bonnefoy, M., see Revel, F. (2) 553-560

Borghi, R., see Monacelli, F. (1) 89-95

Bouleau, S. and H. Tricoire, Drosophila Models of Alzheimer's Disease: Advances, Limits, and Perspectives (4) 1015-1038

Bouter, Y., see Meißner, J.N. (2) 471-482

Bouvier, D.S. and K.K. Murai, Synergistic Actions of Microglia and Astrocytes in the Progression of Alzheimer's Disease (4) 1001-1014

Bozsó, Z., see Varga, E. (2) 449-456

Bramanti, P., see Vecchio, F. (3) 745-756

Brédart, S., see Moyse, E. (2) 631-638

Bresell, A., see Sun, Y. (4) 1061-1076

Brita, A.C., see Silveri, M.C. (2) 483-494

Broeck, B.V., see Struyfs, H. (3) 813-822

Broeckhoven, C.V., see Struyfs, H. (3) 813-822

Brommelhoff, J.A. and D.L. Sultzer, Brain Structure and Function Related to Depression in Alzheimer's Disease: Contributions from Neuroimaging Research (3) 689-703

Brown, E.E., see Chung, J.K. (3) 907-919

Bruggink, K.A., see Jongbloed, W. (1) 35-43

Buissionnière, A., see Baudron, C.R. (4) 1045-1050

Bun, S., C. Ikejima, J. Kida, A. Yoshimura, A.J. Lebowitz, T. Kakuma and T. Asada, A Combination of Supplements May Reduce the Risk of Alzheimer's Disease in Elderly Japanese with Normal Cognition (1) 15-25

Buratti, L., S. Balestrini, C. Altamura, G. Viticchi, L. Falsetti, S. Luzzi, L. Provinciali, F. Vernieri and M. Silvestrini, Markers for the Risk of Progression from Mild Cognitive Impairment to Alzheimer's Disease (3) 883-890

Burns, J.M., see Watts, A. (1) 187-193

Butters, M.A., see Koenig, A.M. (1) 175-185

Byrro, R.M.D., see Miranda, L.F.J.R. (2) 609-620

Byun, J.-I., see Lee, D.Y. (3) 837-849

Cacciari, C., see Di Paola, M. (4) 1097-1108

Caffarra, P., see Gardini, S. (2) 457-470

Cain, K., see Pan, C. (3) 709-719

Calderón-Garcidueñas, L., A. Mora-Tiscareño, M. Franco-Lira, H. Zhu, Z. Lu, E. Solorio, R. TorresJardón and A. D'Angiulli, Decreases in Short
Term Memory, IQ, and Altered Brain Metabolic Ratios in Urban Apolipoprotein $\varepsilon 4$ Children Exposed to Air Pollution (3) 757-770

Calero, M., see Lovestone, S. (1) 75-88

Calero, M., see Olazarán, J. (4) 1157-1173

Callahan, C.M., see Nho, K. (4) 1197-1206

Caltagirone, C., see Di Paola, M. (4) 1097-1108

Cammarata, S., see Monacelli, F. (1) 89-95

Campbell, J., see Leandri, M. (3) 705-707

Campbell, S.N., C. Zhang, A.D. Roe, N. Lee, K.U. Lao, L. Monte, M.C. Donohue and R.A. Rissman, Impact of CRFR1 Ablation on Amyloid- $\beta$ Production and Accumulation in a Mouse Model of Alzheimer's Disease (4) 1175-1184

Caramelli, P., see Miranda, L.F.J.R. (2) 609-620

Caravaggio, F., see Chung, J.K. (3) 907-919

Carl, S.M., see Wilkins, H.M. (1) 305-318

Carriba, P., see Moreno-Grau, S. (2) 621-629

Carter, S.F., see Leuzy, A. (4) 1077-1088

Carvalho, C., see Sena, C.M. (1) 127-138

Casamenti, F., C. Grossi, S. Rigacci, D. Pantano, I. Luccarini and M. Stefani, Oleuropein Aglycone: A Possible Drug against Degenerative Conditions. In Vivo Evidence of its Effectiveness against Alzheimer's Disease (3) 679-688

Casanova, J.P., see Muñiz, R. (1) 295-304

Cha, R.H., see Vassilaki, M. (4) 1237-1245

Chakravarty, M.M., see Chung, J.K. (3) 907-919

Chalmers, K., see Hull, J. (3) 891-905

Chambonnier, L., see Baudron, C.R. (4) 1045-1050

Champagne-Lavau, M., see Moreau, N. (2) 581-597

Charidimou, A., see Lee, D. (4) 1207-1222

Chen, C.L.H., see Cheung, C.Y. (1) 45-56

Chen, S., see Wang, Y. (4) 1185-1195

Cheung, C.Y., Y.T. Ong, S. Hilal, M.K. Ikram, S. Low, Y.L. Ong, N. Venketasubramanian, P. Yap, D. Seow, C.L.H. Chen and T.Y. Wong, Retinal Ganglion Cell Analysis Using High-Definition Optical Coherence Tomography in Patients with Mild Cognitive Impairment and Alzheimer's Disease (1) $45-56$

Chiarelli, M.V.M., see Silveri, M.C. (2) 483-494

Chiotis, K., see Leuzy, A. (4) 1077-1088

Cho, Y., see Baudron, C.R. (4) 1045-1050

Choi, B.-O., see Ye, B.S. (2) 599-608

Choi, S.H., see Ye, B.S. (2) 599-608

Chong, S.A., see Subramaniam, M. (4) 1127-1138

Chow, T.W., see Chung, J.K. (3) 907-919

Chu, K., see Lee, D.Y. (3) 837-849

Chua, B.Y., see Subramaniam, M. (4) 1127-1138

Chua, H.C., see Subramaniam, M. (4) 1127-1138 
Chung, J.K., E. Plitman, S. Nakajima, T.W. Chow, M.M. Chakravarty, F. Caravaggio, P. Gerretsen, E.E. Brown, Y. Iwata, B.H. Mulsant, A. GraffGuerrero and for the Alzheimer's Disease Neuroimaging Initiative, Lifetime History of Depression Predicts Increased Amyloid- $\beta$ Accumulation in Patients with Mild Cognitive Impairment (3) 907919

Colin, J.C., see Pinçon, A. (1) 195-204

Comella, J.X., see Moreno-Grau, S. (2) 621-629

Conway, M., see Hull, J. (3) 891-905

Cook, D.G., see Meeker, K.D. (2) 509-520

Coppus, A.M.W., see Naudé, P.J.W. (3) 733-743

Corbier, C., see Pinçon, A. (1) 195-204

Craig, D., see Williams, M.A. (2) 369-372

Cretin, B., L. Di Bitonto, F. Blanc and E. Magnin, Left Temporal Lobe Epilepsy Revealing Left Posterior Cortical Atrophy Due to Alzheimer's Disease (2) 521-526

Crisi, G., see Gardini, S. (2) 457-470

Cuetos, F., see Gardini, S. (2) 457-470

Cui, D., see Xie, B. (3) 721-731

Cunha, R.A., see Agostinho, P. (2) 329-347

Curcio, G., see Vecchio, F. (3) 745-756

D’Angiulli, A., see Calderón-Garcidueñas, L. (3) 757770

da Cruz e Silva, O.A.B., see Oliveira, J.M. (2) 495-507

Dam, D.V., see Naudé, P.J.W. (3) 733-743

Darst, B.F., R.L. Koscik, B.P. Hermann, A.L. Rue, M.A. Sager, S.C. Johnson and C.D. Engelman, Heritability of Cognitive Traits Among Siblings with a Parental History of Alzheimer's Disease (4) 1149-1155

De Deyn, P.P., see Naudé, P.J.W. (3) 733-743

De Deyn, P.P., see Niemantsverdriet, E. (4) 1039-1043

De Deyn, P.P., see Struyfs, H. (3) 813-822

De Luca, M., see Silveri, M.C. (2) 483-494

Dean, R.A., see Lachno, D.R. (2) 527-542

Degen, C., see Kowoll, M.E. (4) 1257-1268

Deguchi, K., see Matsuzono, K. (3) 771-780

Dekker, A.D., see Naudé, P.J.W. (3) 733-743

DeKosky, S.T., see Wang, X. (2) 651-658

del Ser, T., see Lovestone, S. (1) 75-88

del Ser, T., see Muñiz, R. (1) 295-304

DeLozier, I.J., see Koenig, A.M. (1) 175-185

Demirci, F.Y., see Wang, X. (2) 651-658

Denkinger, M.D., see Nagel, G. (4) 1119-1126

Derrien, A., see Pinçon, A. (1) 195-204

Di Bitonto, L., see Cretin, B. (2) 521-526

Di Paola, M., O. Phillips, M.D. Orfei, F. Piras, C.
Cacciari, C. Caltagirone and G. Spalletta, Corpus Callosum Structure is Topographically Correlated with the Early Course of Cognition and Depression in Alzheimer's Disease (4) 1097-1108

DiFrancesco, J.C., see Boncoraglio, G.B. (2) 363-367

Ding, J., see Wang, Y. (4) 1185-1195

Dlugaj, M., A. Winkler, N. Dragano, S. Moebus, K.-H. Jöckel, R. Erbel and C. Weimar on behalf of the Heinz Nixdorf Recall Study Investigative Group, Depression and Mild Cognitive Impairment in the General Population: Results of the Heinz Nixdorf Recall Study (1) 159-174

Dobson, R., see Khondoker, M. (3) 851-864

Dong, X., see Qin, W. (1) 245-252

Donohue, M.C., see Campbell, S.N. (4) 1175-1184

Doshanjh, L., see Remington, R. (2) 395-405

Dragano, N., see Dlugaj, M. (1) 159-174

Drai, J., see Revel, F. (2) 553-560

Drubach, D.A., see Whitwell, J.L. (4) 1109-1117

Dubois, B., see Lovestone, S. (1) 75-88

Dubus, P., see Baudron, C.R. (4) 1045-1050

Duffy, J.R., see Whitwell, J.L. (4) 1109-1117

Durbin, T., see Hsu, P.J. (1) 27-33

Eckerström, C., see Rolstad, S. (3) 873-881

Ecochard, R., see Revel, F. (2) 553-560

Efthimiopoulos, S., see Kyratzi, E. (1) 147-157

Eisel, U.L.M., see Naudé, P.J.W. (3) 733-743

Elahi, S., A.H. Bachman, S.H. Lee, J.J. Sidtis, B.A. Ardekani and for the Alzheimer's Disease Neuroimaging Initiative, Corpus Callosum Atrophy Rate in Mild Cognitive Impairment and Prodromal Alzheimer's Disease (3) 921-931

El-Fetoh, N.A., see Khedr, E. (1) 117-126

Eng, G.K., see Subramaniam, M. (4) 1127-1138

Engelborghs, S., see Niemantsverdriet, E. (4) 10391043

Engelborghs, S., see Struyfs, H. (3) 813-822

Engelman, C.D., see Darst, B.F. (4) 1149-1155

Erbel, R., see Dlugaj, M. (1) 159-174

Erk, S., see Meiberth, D. (1) 139-146

Eslinger, P.J., see Vasavada, M.M. (3) 947-958

Espinosa, A., see Moreno-Grau, S. (2) 621-629

Evans, A., see Wang, X. (3) 781-795

Evert, B.A., see Lachno, D.R. (2) 527-542

Falsetti, L., see Buratti, L. (3) 883-890

Farina, L., see Boncoraglio, G.B. (2) 363-367

Farlow, M.R., see Nho, K. (4) 1197-1206

Farquhar, R., see Olazarán, J. (4) 1157-1173

Farrer, L.A., see Inzelberg, R. (3) 865-871 
Fasano, F., see Gardini, S. (2) 457-470

Fawi, G., see Khedr, E. (1) 117-126

Feingold, E., see Wang, X. (2) 651-658

Fénelon, V., see Baudron, C.R. (4) 1045-1050

Fernandes, R., see Sena, C.M. (1) 127-138

Fernández, M.V., see Moreno-Grau, S. (2) 621-629

Fernández-Montoya, J. and M. Pérez, Cathepsin D in a

Murine Model of Frontotemporal Dementia with

Parkinsonism-Linked to Chromosome 17 (1) 1-14

Ferrante, I., see Silveri, M.C. (2) 483-494

Ferrer, I., see Armand-Ugón, M. (2) 407-421

Festoff, B.W., see Wilkins, H.M. (1) 305-318

Feyen, B.F.E., see Niemantsverdriet, E. (4) 1039-1043

Fishman, P., see Remington, R. (2) 395-405

Foroud, T.M., see Nho, K. (4) 1197-1206

Frades-Payo, B., see Olazarán, J. (4) 1157-1173

Franco-Lira, M., see Calderón-Garcidueñas, L. (3) 757770

Frank-García, A., see Olazarán, J. (4) 1157-1173

Fransen, E., see Struyfs, H. (3) 813-822

Friedland, R.P., Mechanisms of Molecular Mimicry

Involving the Microbiota in Neurodegeneration

(2) 349-362

Friedland, R.P., see Inzelberg, R. (3) 865-871

Fujiwara, K., see Ito, K. (2) 543-552

Fukuyama, H., see Ito, K. (2) 543-552

Fülöp, L., see Varga, E. (2) 449-456

Galas, M.-C., see Špolcová, A. (3) 823-835

Galasko, D., see Pan, C. (3) 709-719

Gao, S., see Nho, K. (4) 1197-1206

Gao, Y., see Qin, W. (1) 245-252

Gardini, S., A. Venneri, F. Sambataro, F. Cuetos, F. Fasano, M. Marchi, G. Crisi and P. Caffarra, Increased Functional Connectivity in the Default Mode Network in Mild Cognitive Impairment: A Maladaptive Compensatory Mechanism Associated with Poor Semantic Memory Performance (2) 457-470

Geda, Y.E., see Vassilaki, M. (4) 1237-1245

Geerlings, M.I., see Wisse, L.E.M. (4) 1247-1256

Georges, A., see Pinçon, A. (1) 195-204

Gerretsen, P., see Chung, J.K. (3) 907-919

Giaccone, G., see Boncoraglio, G.B. (2) 363-367

Giambattistelli, F., see Vecchio, F. (3) 745-756

Giese, A., see Baudron, C.R. (4) 1045-1050

Gil, R., see Arroyo-Anlló, E.M. (1) 205-216

Gilbert, T., see Revel, F. (2) 553-560

Gil-de-Gómez, L., see Olazarán, J. (4) 1157-1173

Gill, D.J., see Vasavada, M.M. (3) 947-958

Ginghina, C., see Pan, C. (3) 709-719

Gladis, S., see Kowoll, M.E. (4) 1257-1268
Goeman, J., see Niemantsverdriet, E. (4) 1039-1043

Gomes, K.B., see Miranda, L.F.J.R. (2) 609-620

Gómez-Carrillo, B., see Lovestone, S. (1) 75-88

Gordon, R., see Vorobyov, V. (1) 217-233

Gozes, I., A. Yeheskel and M. Pasmanik-Chor, Activity-Dependent Neuroprotective Protein (ADNP):

A Case Study for Highly Conserved ChordataSpecific Genes Shaping the Brain and Mutated in Cancer (1) 57-73

Graff-Guerrero, A., see Chung, J.K. (3) 907-919

Graff-Guerrero, A., see Shinagawa, S. (1) 283-293

Grant, W.B., see Perrone, L. (3) 965-979

Gray, N.E., H. Sampath, J.A. Zweig, J.F. Quinn and A. Soumyanath, Centella asiatica Attenuates Amyloid- $\beta$-Induced Oxidative Stress and Mitochondrial Dysfunction (3) 933-946

Grossi, C., see Casamenti, F. (3) 679-688

Grundke-Iqbal, I., see Wang, X. (2) 423-435

Guan, H., see Yang, H. (3) 797-811

Guevara, J., see Luna-Muñoz, J. (2) 325-327

Guisasola, M.C., see Olazarán, J. (4) 1157-1173

Gunter, J.L., see Whitwell, J.L. (4) 1109-1117

Haluzík, M., see Špolcová, A. (3) 823-835

Han, H.J., see Ye, B.S. (2) 599-608

Han, S.-H., see Moon, Y. (4) 1089-1096

Han, S.-H., see Ye, B.S. (2) 599-608

Harding, J.W., see Wright, J.W. (4) 985-1000

Haughton, D., see Williams, M.A. (2) 369-372

He, Y., see Wang, X. (3) 781-795

He, Y., see Wang, X. (3) 781-795

Hendrie, H.C., see Nho, K. (4) 1197-1206

Heneka, M.T., see Meiberth, D. (1) 139-146

Heng, D., see Subramaniam, M. (4) 1127-1138

Henriques, A.G., see Oliveira, J.M. (2) 495-507

Herbolsheimer, F., see Nagel, G. (4) 1119-1126

Hermann, B.P., see Darst, B.F. (4) 1149-1155

Hernández, I., see Moreno-Grau, S. (2) 621-629

Hezwani, M., see Hull, J. (3) 891-905

Hia, S.B., see Subramaniam, M. (4) 1127-1138

Hilal, S., see Cheung, C.Y. (1) 45-56

Hindy, M.E., see Hull, J. (3) 891-905

Hishikawa, N., see Matsuzono, K. (3) 771-780

Höglund, K., see Sun, Y. (4) 1061-1076

Holubová, M., see Špolcová, A. (3) 823-835

Honea, R.A., see Watts, A. (1) 187-193

Horgusluoglu, E., see Nho, K. (4) 1197-1206

Hsu, P.J., H. Shou, T. Benzinger, D. Marcus, T. Durbin, J.C. Morris and Y.I. Sheline, Amyloid Burden in Cognitively Normal Elderly is Associated with Preferential Hippocampal Subfield Volume Loss (1) $27-33$ 
Hu, X., see Meiberth, D. (1) 139-146

Huang, J., see Zheng, N. (2) 659-671

Huang, W., see Subramaniam, M. (4) 1127-1138

Huang, Y., Z. Wu and B. Zhou, hSOD1 Promotes Tau Phosphorylation and Toxicity in the Drosophila Model (1) 235-244

Huguet, M., see Pinçon, A. (1) 195-204

Hull, J., V. Patel, M.E. Hindy, C. Lee, E. Odeleye, M. Hezwani, S. Love, P. Kehoe, K. Chalmers and M. Conway, Regional Increase in the Expression of the BCAT Proteins in Alzheimer's Disease Brain: Implications in Glutamate Toxicity (3) 891-905 Hüll, M., see Lovestone, S. (1) 75-88

Huppertz, H.-J., see Lovestone, S. (1) 75-88

Hutfles, L., see Watts, A. (1) 187-193

Ikeda, M., see Ito, K. (2) 543-552

Ikejima, C., see Bun, S. (1) 15-25

Ikram, M.K., see Cheung, C.Y. (1) 45-56

Ingrand, P., see Arroyo-Anlló, E.M. (1) 205-216

Inlow, M.H., see Nho, K. (4) 1197-1206

Inzelberg, R., M. Massarwa, E. Schechtman, R. Strugatsky, L.A. Farrer and R.P. Friedland, Estimating the Risk for Conversion from Mild Cognitive Impairment to Alzheimer's Disease in an Elderly Arab Community (3) 865-871

Iqbal, K., see Wang, X. (2) 423-435

Ishii, K., see Ito, K. (2) 543-552

Ishii, K., see Ito, K. (2) 543-552

Ito, K., H. Fukuyama, M. Senda, K. Ishii, K. Maeda, Y. Yamamoto, Y. Ouchi, K. Ishii, A. Okumura, K. Fujiwara, T. Kato, Y. Arahata, Y. Washimi, Y. Mitsuyama, K. Meguro, M. Ikeda and SEAD-J Study Group, Prediction of Outcomes in Mild Cognitive Impairment by Using ${ }^{18}$ F-FDG-PET: A Multicenter Study (2) 543-552

Iwata, Y., see Chung, J.K. (3) 907-919

Jack Jr., C.R., see Whitwell, J.L. (4) 1109-1117

Jacobi, H., see Meiberth, D. (1) 139-146

Jeon, D., see Lee, D.Y. (3) 837-849

Jessen, F., see Meiberth, D. (1) 139-146

Jeyagurunathana, A., see Subramaniam, M. (4) $1127-$ 1138

Jia, J., see Qin, W. (1) 245-252

Jia, X., see Qin, W. (1) 245-252

Jingami, N., M. Asada-Utsugi, K. Uemura, R. Noto, M. Takahashi, A. Ozaki, T. Kihara, T. Kageyama, R. Takahashi, S. Shimohama and A. Kinoshita, Idiopathic Normal Pressure Hydrocephalus has a Different Cerebrospinal Fluid Biomarker Profile from Alzheimer's Disease (1) 109-115
Jöckel, K.-H., see Dlugaj, M. (1) 159-174

Johansson, B., see Rolstad, S. (3) 873-881

Johnson, S.C., see Darst, B.F. (4) 1149-1155

Jongbloed, W., K.A. Bruggink, M.I. Kester, P.-J. Visser, P. Scheltens, M.A. Blankenstein, M.M. Verbeek, C.E. Teunissen and R. Veerhuis, Amyloid- $\beta$ Oligomers Relate to Cognitive Decline in Alzheimer's Disease (1) 35-43

Josephs, K.A., see Whitwell, J.L. (4) 1109-1117

Juhász, G., see Varga, E. (2) 449-456

Jung, K.-H., see Lee, D.Y. (3) 837-849

Jung, K.-Y., see Lee, D.Y. (3) 837-849

Kageyama, T., see Jingami, N. (1) 109-115

Kakuma, T., see Bun, S. (1) 15-25

Kamboh, M.I., see Wang, X. (2) 651-658

Kantarci, K., see Whitwell, J.L. (4) 1109-1117

Kaptsov, V., see Vorobyov, V. (1) 217-233

Karunanayaka, P., see Vasavada, M.M. (3) 947-958

Kato, T., see Ito, K. (2) 543-552

Kehoe, P., see Hull, J. (3) 891-905

Kester, M.I., see Jongbloed, W. (1) 35-43

Khedr, E., G. Fawi, M.A.A. Abbas, T.A. Mohammed, N.A. El-Fetoh, G.A. Attar, M. Noaman and A.F. Zaki, Prevalence of Mild Cognitive Impairment and Dementia among the Elderly Population of Qena Governorate, Upper Egypt: A CommunityBased Study (1) 117-126

Khondoker, M., S. Newhouse, E. Westman, J.-S. Muehlboeck, P. Mecocci, B. Vellas, M. Tsolaki, I. Kłoszewska, H. Soininen, S. Lovestone, R. Dobson, A. Simmons and for the AddNeuroMed consortium and for the Alzheimer's Disease Neuroimaging Initiative, Linking Genetics of Brain Changes to Alzheimer's Disease: Sparse Whole Genome Association Scan of Regional MRI Volumes in the ADNI and AddNeuroMed Cohorts (3) $851-864$

Kida, J., see Bun, S. (1) 15-25

Kihara, T., see Jingami, N. (1) 109-115

Kim, E.-J., see Ye, B.S. (2) 599-608

Kim, M., see Lee, D.Y. (3) 837-849

Kim, S., see Nho, K. (4) 1197-1206

Kim, S.H., see Ye, B.S. (2) 599-608

Kim, S.Y., see Ye, B.S. (2) 599-608

Kim, W.C., see Lee, D. (4) 1207-1222

Kinoshita, A., see Jingami, N. (1) 109-115

Kłoszewska, I., see Khondoker, M. (3) 851-864

Knopman, D.S., see Vassilaki, M. (4) 1237-1245

Koek, H.L., see Wisse, L.E.M. (4) 1247-1256

Koenig, A.M., I.J. DeLozier, M.D. Zmuda, M.M. Marron, A.E. Begley, S.J. Anderson, C.F. Reynolds 
III, S.E. Arnold, J.T. Becker and M.A. Butters, Neuropsychological Functioning in the Acute and Remitted States of Late-Life Depression (1) 175185

Koenig, W., see Nagel, G. (4) 1119-1126

Korff, A., see Pan, C. (3) 709-719

Koscik, R.L., see Darst, B.F. (4) 1149-1155

Kowoll, M.E., C. Degen, S. Gladis and J. Schröder, Neuropsychological Profiles and Verbal Abilities in Lifelong Bilinguals with Mild Cognitive Impairment and Alzheimer's Disease (4) 12571268

Kua, J., see Subramaniam, M. (4) 1127-1138

Kuijf, H.J., see Wisse, L.E.M. (4) 1247-1256

Kum Jew, S., see Pamphlett, R. (2) 437-447

Kuo, C.-C., see Zhang, C. (2) 639-650

Kurdakova, A., see Richard, B.C. (4) 1223-1236

Kwon, H., see Moon, Y. (4) 1089-1096

Kyratzi, E., A. Liakos, G. Papadogiannaki and S. Efthimiopoulos, Structural and Regulatory Elements of the Interaction between Amyloid- $\beta$ Protein Precursor and Homer3 (1) 147-157

Labib, W., see Sun, S.-W. (4) 1139-1148

Lachno, D.R., B.A. Evert, K. Maloney, B.A. Willis, J.A. Talbot, M. Vandijck and R.A. Dean, Validation and Clinical Utility of ELISA Methods for Quantification of Amyloid- $\beta$ Peptides in Cerebrospinal Fluid Specimens from Alzheimer's Disease Studies (2) 527-542

LaCross, A., see Berisha, V. (3) 959-963

Ladd, A.C., see Rice, A.C. (4) 1051-1059

Lanhers, M.-C., see Pinçon, A. (1) 195-204

Lao, K.U., see Campbell, S.N. (4) 1175-1184

Larsen, D., see Remington, R. (2) 395-405

Lattanzi, S., S. Luzzi, L. Provinciali and M. Silvestrini, Blood Pressure Variability in Alzheimer's Disease and Frontotemporal Dementia: The Effect on the Rate of Cognitive Decline (2) 387-394

Leandri, M., J. Campbell, L. Molfetta, C. Barbera and M. Tabaton, Relationship Between Balance and Cognitive Performance in Older People (3) 705707

Lebouvier, T., see Sun, Y. (4) 1061-1076

Lebowitz, A.J., see Bun, S. (1) 15-25

Lee, C., see Hull, J. (3) 891-905

Lee, D., W.C. Kim, A. Charidimou and M. Song, A Bird's-Eye View of Alzheimer's Disease Research: Reflecting Different Perspectives of Indexers, Authors, or Citers in Mapping the Field (4) $1207-1222$

Lee, D.Y., J. Moon, S.-T. Lee, K.-H. Jung, D.-K. Park,
J.-S. Yoo, J.-S. Sunwoo, J.-I. Byun, J.-W. Shin, D. Jeon, K.-Y. Jung, M. Kim, S.K. Lee and K. Chu, Distinct Expression of Long Non-Coding RNAs in an Alzheimer's Disease Model (3) 837-849

Lee, J.-M., see Moon, Y. (4) 1089-1096

Lee, N., see Campbell, S.N. (4) 1175-1184

Lee, S.H., see Elahi, S. (3) 921-931

Lee, S.K., see Lee, D.Y. (3) 837-849

Lee, S.P., see Subramaniam, M. (4) 1127-1138

Lee, S.-T., see Lee, D.Y. (3) 837-849

Leemans, A., see Wisse, L.E.M. (4) 1247-1256

Lehours, P., see Baudron, C.R. (4) 1045-1050

León, T., see Lovestone, S. (1) 75-88

Leuzy, A., S.F. Carter, K. Chiotis, O. Almkvist, A. Wall and A. Nordberg, Concordance and Diagnostic Accuracy of $\left[{ }^{11} \mathrm{C}\right] \mathrm{PIB}$ PET and Cerebrospinal Fluid Biomarkers in a Sample of Patients with Mild Cognitive Impairment and Alzheimer's Disease (4) 1077-1088

Li, C., see Zheng, N. (2) 659-671

Li, D., see Qin, W. (1) 245-252

Li, G., see Pan, C., (3) 709-719

Li, H., see Wang, X. (3) 781-795

Li, J., see Sun, Y. (1) 269-282

Li, Y., see Qin, W. (1) 245-252

Liakos, A., see Kyratzi, E. (1) 147-157

Liang, H.-F., see Sun, S.-W. (4) 1139-1148

Lima, T.O.L., see Miranda, L.F.J.R. (2) 609-620

Linseman, D.A., see Wilkins, H.M. (1) 305-318

Liperoti, R., see Silveri, M.C. (2) 483-494

Liss, J., see Berisha, V. (3) 959-963

Liu, J., see Zhang, H. (2) 561-580

Liu, Z., see Xie, B. (3) 721-731

Liu, Z., see Yang, H. (3) 797-811

López, J., see Moreno-Grau, S. (2) 621-629

Lopez, O., see Wang, X. (2) 651-658

López-Arrieta, J., see Moreno-Grau, S. (2) 621-629

Love, S., see Hull, J. (3) 891-905

Lovestone, S., M. Boada, B. Dubois, M. Hüll, J.O. Rinne, H.-J. Huppertz, M. Calero, M.V. Andrés, B. Gómez-Carrillo, T. León and T. del Ser for the ARGO investigators, A Phase II Trial of Tideglusib in Alzheimer's Disease (1) 75-88

Lovestone, S., see Khondoker, M. (3) 851-864

Low, S., see Cheung, C.Y. (1) 45-56

Lowe, V.J., see Whitwell, J.L. (4) 1109-1117

Lu, W., see Sun, Y. (1) 269-282

Lu, Z., see Calderón-Garcidueñas, L. (3) 757-770

Luccarini, I., see Casamenti, F. (3) 679-688

Ludolph, A.C., see Nagel, G. (4) 1119-1126

Luijten, P.R., see Wisse, L.E.M. (4) 1247-1256

Luna-Muñoz, J., G. Perry and J. Guevara, Raúl Mena: 
1953-2014 (2) 325-327

Lundt, E.S., see Whitwell, J.L. (4) 1109-1117

Luo, Y., see Remington, R. (2) 395-405

Luzzi, S., see Buratti, L. (3) 883-890

Luzzi, S., see Lattanzi, S. (2) 387-394

Machulda, M.M., see Whitwell, J.L. (4) 1109-1117

Macrez, N., see Baudron, C.R. (4) 1045-1050

Maeda, K., see Ito, K. (2) 543-552

Magadi, H., see Subramaniam, M. (4) 1127-1138

Magnin, E., see Cretin, B. (2) 521-526

Mahendran, R., see Subramaniam, M. (4) 1127-1138

Makarova, E., see Vorobyov, V. (1) 217-233

Malaplate-Armand, C., see Pinçon, A. (1) 195-204

Maletínská, L., see Špolcová, A. (3) 823-835

Malladi, S., see Subramaniam, M. (4) 1127-1138

Maloney, K., see Lachno, D.R. (2) 527-542

Mammarella, F., see Silveri, M.C. (2) 483-494

Manzano-Palomo, S., see Olazarán, J. (4) 1157-1173

Marchi, M., see Gardini, S. (2) 457-470

Marcus, D., see Hsu, P.J. (1) 27-33

Marín, J., see Moreno-Grau, S. (2) 621-629

Marín-Muñoz, J., see Olazarán, J. (4) 1157-1173

Marron, M.M., see Koenig, A.M. (1) 175-185

Martin, J.-J., see Niemantsverdriet, E. (4) 1039-1043

Martinez-Almoyna, L., see Moreau, N. (2) 581-597

Martins, F., see Oliveira, J.M. (2) 495-507

Massarwa, M., see Inzelberg, R. (3) 865-871

Matoso, R.O., see Miranda, L.F.J.R. (2) 609-620

Matsuzono, K., N. Hishikawa, Y. Ohta, T. Yamashita, K. Deguchi, Y. Nakano and K. Abe, Combination Therapy of Cholinesterase Inhibitor (Donepezil or Galantamine) plus Memantine in the Okayama Memantine Study (3) 771-780

Mauleón, A., see Moreno-Grau, S. (2) 621-629

McCrone, P., see Subramaniam, M. (4) 1127-1138

McDonald, B.C., see Nho, K. (4) 1197-1206

Meabon, J.S., see Meeker, K.D. (2) 509-520

Mecocci, P., see Khondoker, M. (3) 851-864

Meeker, K.D., J.S. Meabon and D.G. Cook, Partial Loss of the Glutamate Transporter GLT-1 Alters Brain Akt and Insulin Signaling in a Mouse Model of Alzheimer's Disease (2) 509-520

Mégraud, F., see Baudron, C.R. (4) 1045-1050

Meguro, K., see Ito, K. (2) 543-552

Meiberth, D., L. Scheef, S. Wolfsgruber, H. Boecker, W. Block, F. Träber, S. Erk, M.T. Heneka, H. Jacobi, A. Spottke, H. Walter, M. Wagner, X. Hu and F. Jessen, Cortical Thinning in Individuals with Subjective Memory Impairment (1) 139-146

Meißner, J.N., Y. Bouter and T.A. Bayer, Neuron Loss and Behavioral Deficits in the TBA42 Mouse Model Expressing N-Truncated Pyroglutamate Amyloid- $\beta_{3-42}$ (2) 471-482

Mercken, M., see Struyfs, H. (3) 813-822

Mielke, M.M., see Vassilaki, M. (4) 1237-1245

Mikulášková, B., see Špolcová, A. (3) 823-835

Miller, B.L., see Shinagawa, S. (1) 283-293

Mimura, M., see Shinagawa, S. (1) 283-293

Min, B., see Qin, W. (1) 245-252

Miraglia, F., see Vecchio, F. (3) 745-756

Miranda, L.F.J.R., K.B. Gomes, J.N. Silveira, G.A. Pianetti, R.M.D. Byrro, P.R.H. Peles, F.H. Pereira, T.R. Santos, A.G. Assini, V.V. Ribeiro, P.A.L. Tito, R.O. Matoso, T.O.L. Lima, E.N. Moraes and P. Caramelli, Predictive Factors of Clinical Response to Cholinesterase Inhibitors in Mild and Moderate Alzheimer's Disease and Mixed Dementia: A One-Year Naturalistic Study (2) 609-620

Mitsuyama, Y., see Ito, K. (2) 543-552

Moebus, S., see Dlugaj, M. (1) 159-174

Moghadam, S.H., see Zhang, C. (2) 639-650

Mohammed, T.A., see Khedr, E. (1) 117-126

Molfetta, L., see Leandri, M. (3) 705-707

Monacelli, F., R. Borghi, S. Cammarata, A. Nencioni, A. Piccini, M. Tabaton and P. Odetti, Amnestic Mild Cognitive Impairment and Conversion to Alzheimer's Disease: Insulin Resistance and Glycoxidation as Early Biomarker Clusters (1) 89-95

Monte, L., see Campbell, S.N. (4) 1175-1184

Monte, L., see Zhang, C. (2) 639-650

Montine, T.J., see Pan, C., (3) 709-719

Moon, J., see Lee, D.Y. (3) 837-849

Moon, W.-J., see Moon, Y. (4) 1089-1096

Moon, Y., W.-J. Moon, H. Kwon, J.-M. Lee and S.-H. Han, Vitamin D Deficiency Disrupts Neuronal Integrity in Cognitively Impaired Patients (4) 1089-1096

Moraes, E.N., see Miranda, L.F.J.R. (2) 609-620

Mora-Tiscareño, A., see Calderón-Garcidueñas, L. (3) 757-770

Moreau, N., S. Rauzy, B. Bonnefoi, L. Renié, L. Martinez-Almoyna, F. Viallet and M. Champagne-Lavau, Different Patterns of Theory of Mind Impairment in Mild Cognitive Impairment (2) 581-597

Moreira, P.I., see Sena, C.M. (1) 127-138

Moreno, J., see Armand-Ugón, M. (2) 407-421

Moreno-Grau, S., B. Barneda, P. Carriba, J. Marín, O. Sotolongo-Grau, I. Hernández, M. Rosende-Roca, A. Mauleón, L. Vargas, A. Espinosa, M. Alegret, O. Rodriguez, G. Ortega, M.V. Fernández, J. López-Arrieta, L. Tárraga, M. Boada, C. Antúnez, 
J. López, A. Ruiz and J.X. Comella, Evaluation of Candidate Genes Related to Neuronal Apoptosis in Late-Onset Alzheimer's Disease (2) 621-629

Morlán-Gracia, L., see Olazarán, J. (4) 1157-1173

Morrell, C. see Remington, R. (2) 395-405

Morris, J.C., see Hsu, P.J. (1) 27-33

Moyse, E., C. Bastin, E. Salmon and S. Brédart, Impairment of Age Estimation from Faces in Alzheimer's Disease (2) 631-638

Muehlboeck, J.-S., see Khondoker, M. (3) 851-864

Mulsant, B.H., see Chung, J.K. (3) 907-919

Muñiz, R., C.M. Serra, B. Reisberg, J.M. Rojo, T. del Ser, J.P. Casanova and J. Olazarán, CognitiveMotor Intervention in Alzheimer's Disease: LongTerm Results from the Maria Wolff Trial (1) 295 304

Murai, K.K., see Bouvier, D.S. (4) 1001-1014

Na, D.L., see Ye, B.S. (2) 599-608

Nagel, G., F. Herbolsheimer, M. Riepe, T. Nikolaus, M.D. Denkinger, R. Peter, G. Weinmayr, D. Rothenbacher, W. Koenig, A.C. Ludolph, C.A.F. von Arnim and the ActiFE Study group, Serum Vitamin D Concentrations and Cognitive Function in a Population-Based Study among Older Adults in South Germany (4) 1119-1126

Nagelová, V., see Špolcová, A. (3) 823-835

Nakajima, S., see Chung, J.K. (3) 907-919

Nakajima, S., see Shinagawa, S. (1) 283-293

Nakano, Y., see Matsuzono, K. (3) 771-780

Nakayama, K., see Shinagawa, S. (1) 283-293

Naudé, P.J.W., A.D. Dekker, A.M.W. Coppus, Y. Vermeiren, U.L.M. Eisel, C.M. van Duijn, D.V. Dam and P.P. De Deyn, Serum NGAL is Associated with Distinct Plasma Amyloid- $\beta$ Peptides According to the Clinical Diagnosis of Dementia in Down Syndrome (3) 733-743

Neau, J.-P., see Arroyo-Anlló, E.M. (1) 205-216

Nencioni, A., see Monacelli, F. (1) 89-95

Newhouse, S., see Khondoker, M. (3) 851-864

Ng, L.L., see Subramaniam, M. (4) 1127-1138

Nho, K., V.K. Ramanan, E. Horgusluoglu, S. Kim, M.H. Inlow, S.L. Risacher, B.C. McDonald, M.R. Farlow, T.M. Foroud, S. Gao, C.M. Callahan, H.C. Hendrie, A.B. Niculescu, A.J. Saykin and for the Alzheimer's Disease Neuroimaging Initiative (ADNI), Comprehensive Gene- and PathwayBased Analysis of Depressive Symptoms in Older Adults (4) 1197-1206

Niculescu, A.B., see Nho, K. (4) 1197-1206

Niemantsverdriet, E., B.F.E. Feyen, N.L. Bastard,
J.-J. Martin, J. Goeman, P.P. De Deyn and S. Engelborghs, Overdiagnosing Vascular Dementia using Structural Brain Imaging for Dementia Work-Up (4) 1039-1043

Nikolaus, T., see Nagel, G. (4) 1119-1126

Nishioka, C., C. Poh and S.-W. Sun, for the Alzheimer's Disease Neuroimaging Initiative, Diffusion Tensor Imaging Reveals Visual Pathway Damage in Patients with Mild Cognitive Impairment and Alzheimer's Disease (1) 97-107

Nishioka, C., see Sun, S.-W. (4) 1139-1148

Noaman, M., see Khedr, E. (1) 117-126

Nordberg, A., see Leuzy, A. (4) 1077-1088

Noto, R., see Jingami, N. (1) 109-115

Odeleye, E., see Hull, J. (3) 891-905

Odetti, P., see Monacelli, F. (1) 89-95

Ohta, Y., see Matsuzono, K. (3) 771-780

Okumura, A., see Ito, K. (2) 543-552

Olazarán, J., see Muñiz, R. (1) 295-304

Olazarán, J., L. Gil-de-Gómez, A. Rodríguez-Martín, M. Valentí-Soler, B. Frades-Payo, J. Marín-Muñoz, C. Antúnez, A. Frank-García, C. Acedo-Jiménez, L. Morlán-Gracia, R. Petidier-Torregrossa, M.C. Guisasola, F. Bermejo-Pareja, Á. Sánchez-Ferro, D.A. Pérez-Martínez, S. Manzano-Palomo, R. Farquhar, A. Rábano and M. Calero, A BloodBased, 7-Metabolite Signature for the Early Diagnosis of Alzheimer's Disease (4) 1157-1173

Oliveira, C.R., see Agostinho, P. (2) 329-347

Oliveira, C.R., see Sena, C.M. (1) 127-138

Oliveira, J.M., A.G. Henriques, F. Martins, S. Rebelo and O.A.B. da Cruz e Silva, Amyloid- $\beta$ Modulates Both A $\beta P P$ and Tau Phosphorylation (2) 495-507

Olivier, J.L., see Pinçon, A. (1) 195-204

Ong, Y.L., see Cheung, C.Y. (1) 45-56

Ong, Y.T., see Cheung, C.Y. (1) 45-56

Orfei, M.D., see Di Paola, M. (4) 1097-1108

Ortega, G., see Moreno-Grau, S. (2) 621-629

Oster, T., see Pinçon, A. (1) 195-204

Ouchi, Y., see Ito, K. (2) 543-552

Ozaki, A., see Jingami, N. (1) 109-115

Page, R. see Remington, R. (2) 395-405

Pamphlett, R., and S. Kum Jew, Different Populations of Human Locus Ceruleus Neurons Contain Heavy Metals or Hyperphosphorylated Tau: Implications for Amyloid- $\beta$ and Tau Pathology in Alzheimer's Disease (2) 437-447

Pan, C., A. Korff, D. Galasko, C. Ginghina, E. Peskind, G. Li, J. Quinn, T.J. Montine, K. Cain, M. Shi 
and J. Zhang, Diagnostic Values of Cerebrospinal Fluid T-Tau and $A \beta_{42}$ using Meso Scale Discovery Assays for Alzheimer's Disease (3) 709-719

Pang, S., see Subramaniam, M. (4) 1127-1138

Pantano, D., see Casamenti, F. (3) 679-688

Papadogiannaki, G., see Kyratzi, E. (1) 147-157

Parati, E.A., see Boncoraglio, G.B. (2) 363-367

Park, D.-K., see Lee, D.Y. (3) 837-849

Park, K.H., see Ye, B.S. (2) 599-608

Pasmanik-Chor, M., see Gozes, I. (1) 57-73

Passmore, A.P., see Williams, M.A. (2) 369-372

Patel, V., see Hull, J. (3) 891-905

Pchitskaya, E., see Zhang, H. (2) 561-580

Pei, G., see Wang, Y. (4) 1185-1195

Peles, P.R.H., see Miranda, L.F.J.R. (2) 609-620

Peng, Y., see Sun, Y. (1) 269-282

Penke, B., see Varga, E. (2) 449-456

Pereira, A.M., see Sena, C.M. (1) 127-138

Pereira, F.H., see Miranda, L.F.J.R. (2) 609-620

Pérez, M., see Fernández-Montoya, J. (1) 1-14

Pérez-Martínez, D.A., see Olazarán, J. (4) 1157-1173

Perrone, L. and W.B. Grant, Observational and Ecological Studies of Dietary Advanced Glycation End Products in National Diets and Alzheimer's Disease Incidence and Prevalence (3) 965-979

Perry, G., see Luna-Muñoz, J. (2) 325-327

Peskind, E., see Pan, C. (3) 709-719

Peter, R., see Nagel, G. (4) 1119-1126

Petersen, R.C., see Vassilaki, M. (4) 1237-1245

Petersen, R.C., see Whitwell, J.L. (4) 1109-1117

Petidier-Torregrossa, R., see Olazarán, J. (4) 1157-1173

Phillips, O., see Di Paola, M. (4) 1097-1108

Pianetti, G.A., see Miranda, L.F.J.R. (2) 609-620

Piazza, F., see Boncoraglio, G.B. (2) 363-367

Piccini, A., see Monacelli, F. (1) 89-95

Picco, L., see Subramaniam, M. (4) 1127-1138

Pillot, T., see Pinçon, A. (1) 195-204

Pinçon, A., M.H. Thomas, M. Huguet, A. Allouche, J.C. Colin, A. Georges, A. Derrien, M.-C. Lanhers, C. Malaplate-Armand, T. Oster, C. Corbier, T. Pillot, J.L. Olivier and F.T. Yen, Increased Susceptibility of Dyslipidemic LSR ${ }^{+-}$Mice to Amyloid Stress is Associated with Changes in Cortical Cholesterol Levels (1) 195-204

Piras, F., see Di Paola, M. (4) 1097-1108

Pirnik, Z., see Špolcová, A. (3) 823-835

Pliássova, A., see Agostinho, P. (2) 329-347

Plitman, E., see Chung, J.K. (3) 907-919

Plitman, E., see Shinagawa, S. (1) 283-293

Podolski, I., see Vorobyov, V. (1) 217-233

Poh, C., see Nishioka, C. (1) 97-107
Popugaeva, E., see Zhang, H. (2) 561-580

Prince, M., see Subramaniam, M. (4) 1127-1138

Prioni, S., see Boncoraglio, G.B. (2) 363-367

Provinciali, L., see Buratti, L. (3) 883-890

Provinciali, L., see Lattanzi, S. (2) 387-394

Pua, E.P.K., see Susanto, T.A.K. (1) 253-268

Qin, W., X. Jia, F. Wang, X. Zuo, L. Wu, A. Zhou, D. Li, B. Min, C. Wei, Y. Tang, Y. Xing, X. Dong, Q. Wang, Y. Gao, Y. Li and J. Jia, Elevated Plasma Angiogenesis Factors in Alzheimer's Disease (1) 245-252

Quattrocchi, C.C., see Vecchio, F. (3) 745-756

Quinn, J., see Pan, C., (3) 709-719

Quinn, J.F., see Gray, N.E. (3) 933-946

Rábano, A., see Olazarán, J. (4) 1157-1173

Ramanan, V.K., see Nho, K. (4) 1197-1206

Ramanujan, S.A., see Wilkins, H.M. (1) 305-318

Rantalainen, M., see Sun, Y. (4) 1061-1076

Rauzy, S., see Moreau, N. (2) 581-597

Rebelo, S., see Oliveira, J.M. (2) 495-507

Reijmer, Y.D., see Wisse, L.E.M. (4) 1247-1256

Reisberg, B., see Muñiz, R. (1) 295-304

Remington, R., C. Bechtel, D. Larsen, A. Samar, L. Doshanjh, P. Fishman, Y. Luo, K. Smyers, R. Page and C. Morrell and T.B. Shea, A Phase II Randomized Clinical Trial of a Nutritional Formulation for Cognition and Mood in Alzheimer's Disease (2) 395-405

Renié, L., see Moreau, N. (2) 581-597

Revel, F., T. Gilbert, S. Roche, J. Drai, E. Blond, R. Ecochard and M. Bonnefoy, Influence of Oxidative Stress Biomarkers on Cognitive Decline (2) 553-560

Reynolds III, C.F., see Koenig, A.M. (1) 175-185

Rhyner, K.T., see Watts, A. (1) 187-193

Ribeiro, V.V., see Miranda, L.F.J.R. (2) 609-620

Rice, A.C., A.C. Ladd and J.P. Bennett Jr, Postmortem Alzheimer's Disease Hippocampi Show Oxidative Phosphorylation Gene Expression Opposite that of Isolated Pyramidal Neurons (4) 1051-1059

Rice, K.C., see Zhang, C. (2) 639-650

Richard, B.C., A. Kurdakova, S. Baches, T.A. Bayer, S. Weggen and O. Wirths, Gene Dosage Dependent Aggravation of the Neurological Phenotype in the 5XFAD Mouse Model of Alzheimer's Disease (4) 1223-1236

Riepe, M., see Nagel, G. (4) 1119-1126

Riera-Codina, M., see Armand-Ugón, M. (2) 407-421

Rigacci, S., see Casamenti, F. (3) 679-688 
Rinne, J.O., see Lovestone, S. (1) 75-88

Risacher, S.L., see Nho, K. (4) 1197-1206

Rissman, R.A., see Campbell, S.N. (4) 1175-1184

Rissman, R.A., see Zhang, C. (2) 639-650

Roberts, R.O., see Vassilaki, M. (4) 1237-1245

Roche, S., see Revel, F. (2) 553-560

Rodriguez, O., see Moreno-Grau, S. (2) 621-629

Rodríguez-Martín, A., see Olazarán, J. (4) 1157-1173

Roe, A.D., see Campbell, S.N. (4) 1175-1184

Rojo, J.M., see Muñiz, R. (1) 295-304

Rolstad, S., A.I. Berg, C. Eckerström, B. Johansson and A. Wallin, Differential Impact of Neurofilament Light Subunit on Cognition and Functional Outcome in Memory Clinic Patients with and without Vascular Burden (3) 873-881

Rong, X., see Sun, Y. (1) 269-282

Rosende-Roca, M., see Moreno-Grau, S. (2) 621-629

Rossi, P., see Silveri, M.C. (2) 483-494

Rossini, P.M., see Vecchio, F. (3) 745-756

Rothenbacher, D., see Nagel, G. (4) 1119-1126

Rue, A.L., see Darst, B.F. (4) 1149-1155

Ruiz, A., see Moreno-Grau, S. (2) 621-629

Sagayadevan, V., see Subramaniam, M. (4) 1127-1138

Sager, M.A., see Darst, B.F. (4) 1149-1155

Salles, N., see Baudron, C.R. (4) 1045-1050

Salmon, E., see Moyse, E. (2) 631-638

Salter, H. see Sun, Y. (4) 1061-1076

Samar, A., see Remington, R. (2) 395-405

Sambasivam, R., see Subramaniam, M. (4) 1127-1138

Sambataro, F., see Gardini, S. (2) 457-470

Sampath, H., see Gray, N.E. (3) 933-946

Sánchez, A., see Armand-Ugón, M. (2) 407-421

Sánchez-Ferro, Á., see Olazarán, J. (4) 1157-1173

Santos, T.R., see Miranda, L.F.J.R. (2) 609-620

Savoiardo, M., see Boncoraglio, G.B. (2) 363-367

Saykin, A.J., see Nho, K. (4) 1197-1206

Schechtman, E., see Inzelberg, R. (3) 865-871

Scheef, L., see Meiberth, D. (1) 139-146

Scheltens, P., see Jongbloed, W. (1) 35-43

Schröder, J., see Kowoll, M.E. (4) 1257-1268

Scrascia, F., see Vecchio, F. (3) 745-756

Seiça, R.M., see Sena, C.M. (1) 127-138

Sena, C.M., A.M. Pereira, C. Carvalho, R. Fernandes, R.M. Seiça, C.R. Oliveira and P.I. Moreira, Type 2 Diabetes Aggravates Alzheimer's Disease-Associated Vascular Alterations of the Aorta in Mice (1) 127-138

Senda, M., see Ito, K. (2) 543-552

Seng, K.H., see Subramaniam, M. (4) 1127-1138

Sengpiel, F., see Vorobyov, V. (1) 217-233

Seow, D., see Cheung, C.Y. (1) 45-56
Seow, E., see Subramaniam, M. (4) 1127-1138

Serra, C.M., see Muñiz, R. (1) 295-304

Shafie, S., see Subramaniam, M. (4) 1127-1138

Shahwan, S., see Subramaniam, M. (4) 1127-1138

Shea, T.B., see Remington, R. (2) 395-405

Sheline, Y.I., see Hsu, P.J. (1) 27-33

Shen, X., see Wang, Y. (4) 1185-1195

Shi, M., see Pan, C. (3) 709-719

Shimohama, S., see Jingami, N. (1) 109-115

Shin, J.-W., see Lee, D.Y. (3) 837-849

Shinagawa, S., S. Nakajima, E. Plitman, A. Graff-Guerrero, M. Mimura, K. Nakayama and B.L. Miller, Non-Pharmacological Management for Patients with Frontotemporal Dementia: A Systematic Review (1) 283-293

Shou, H., see Hsu, P.J. (1) 27-33

Sidtis, J.J., see Elahi, S. (3) 921-931

Silveira, J.N., see Miranda, L.F.J.R. (2) 609-620

Silveri, M.C., I. Ferrante, A.C. Brita, P. Rossi, R. Liperoti, F. Mammarella, R. Bernabei, M.V.M. Chiarelli and M. De Luca, "The Memory of Beauty" Survives Alzheimer's Disease (but Cannot Help Memory) (2) 483-494

Silvestri, G., see Williams, M.A. (2) 369-372

Silvestrini, M., see Buratti, L. (3) 883-890

Silvestrini, M., see Lattanzi, S. (2) 387-394

Simmons, A., see Khondoker, M. (3) 851-864

Sleegers, K., see Struyfs, H. (3) 813-822

Smyers, K., see Remington, R. (2) 395-405

Soininen, H., see Khondoker, M. (3) 851-864

Solorio, E., see Calderón-Garcidueñas, L. (3) 757-770

Song, M., see Lee, D. (4) 1207-1222

Song, M., see Xie, B. (3) 721-731

Sotolongo-Grau, O., see Moreno-Grau, S. (2) 621-629

Soumyanath, A., see Gray, N.E. (3) 933-946

Spalletta, G., see Di Paola, M. (4) 1097-1108

Špolcová, A., B. Mikulášková, M. Holubová, V. Nagelová, Z. Pirnik, J. Zemenová, M. Haluzík, B. Železná, M.-C. Galas and L. Maletínská, Anorexigenic Lipopeptides Ameliorate Central Insulin Signaling and Attenuate Tau Phosphorylation in Hippocampi of Mice with Monosodium Glutamate-Induced Obesity (3) 823-835

Spottke, A., see Meiberth, D. (1) 139-146

Spychalla, A.J., see Whitwell, J.L. (4) 1109-1117

Stefani, M., see Casamenti, F. (3) 679-688

Stevenson, M., see Williams, M.A. (2) 369-372

Strand, E.A., see Whitwell, J.L. (4) 1109-1117

Streffer, J.R., see Struyfs, H. (3) 813-822

Strugatsky, R., see Inzelberg, R. (3) 865-871

Struyfs, H., B.V. Broeck, M. Timmers, E. Fransen, K. Sleegers, C.V. Broeckhoven, P.P. De Deyn, 
J.R. Streffer, M. Mercken and S. Engelborghs, Diagnostic Accuracy of Cerebrospinal Fluid Amyloid- $\beta$ Isoforms for Early and Differential Dementia Diagnosis (3) 813-822

Subramaniam, M., S.A. Chong, J.A. Vaingankar, E. Abdin, B.Y. Chua, H.C. Chua, G.K. Eng, D. Heng, S.B. Hia, W. Huang, A. Jeyagurunathana, J. Kua, S.P. Lee, R. Mahendran, H. Magadi, S. Malladi, P. McCrone, S. Pang, L. Picco, V. Sagayadevan, R. Sambasivam, K.H. Seng, E. Seow, S. Shafie, S. Shahwan, L.L. Tan, M. Yap, Y. Zhang, L.L. Ng and M. Prince, Prevalence of Dementia in People Aged 60 Years and Above: Results from the WiSE Study (4) 1127-1138

Sui, Y., see Wang, Y. (4) 1185-1195

Sultzer, D.L., see Brommelhoff, J.A. (3) 689-703

Sun, S., see Zhang, H. (2) 561-580

Sun, S.-W., C. Nishioka, W. Labib and H.-F. Liang, Axonal Terminals Exposed to Amyloid- $\beta$ May Not Lead to Pre-Synaptic Axonal Damage (4) 1139-1148

Sun, S.-W., see Nishioka, C. (1) 97-107

Sun, X., see Vasavada, M.M. (3) 947-958

Sun, Y., A. Bresell, M. Rantalainen, K. Höglund, T. Lebouvier, H. Salter and the Alzheimer Disease Neuroimaging Initiative, An Integrated Bioinformatics Approach for Identifying Genetic Markers that Predict Cerebrospinal Fluid Biomarker p-tau ${ }_{181} / \mathrm{A} \beta_{1-42}$ Ratio in ApoE4Negative Mild Cognitive Impairment Patients (4) 1061-1076

Sun, Y., X. Rong, W. Lu, Y. Peng, J. Li, S. Xu, L. Wang and X. Wang, Translational Study of Alzheimer's Disease (AD) Biomarkers from Brain Tissues in A $\beta P P / P S 1$ Mice and Serum of AD Patients (1) 269-282

Sunwoo, J.-S., see Lee, D.Y. (3) 837-849

Susanto, T.A.K., E.P.K. Pua, J. Zhou and for the Alzheimer's Disease Neuroimaging Initiative, Cognition, Brain Atrophy, and Cerebrospinal Fluid Biomarkers Changes from Preclinical to Dementia Stage of Alzheimer's Disease and the Influence of Apolipoprotein E (1) 253-268

Sweet, R.A., see Wang, X. (2) 651-658

Swerdlow, R.H., see Wilkins, H.M. (1) 305-318

Szegedi, V., see Varga, E. (2) 449-456

Tabaton, M., see Leandri, M. (3) 705-707

Tabaton, M., see Monacelli, F. (1) 89-95

Tagliavini, F., see Boncoraglio, G.B. (2) 363-367

Takahashi, M., see Jingami, N. (1) 109-115

Takahashi, R., see Jingami, N. (1) 109-115
Takeuchi, S., see Yang, H. (3) 797-811

Talbot, J.A., see Lachno, D.R. (2) 527-542

Tan, L.L., see Subramaniam, M. (4) 1127-1138

Tang, Y., see Qin, W. (1) 245-252

Tárraga, L., see Moreno-Grau, S. (2) 621-629

ter Telgte, A., see Wisse, L.E.M. (4) 1247-1256

Teunissen, C.E., see Jongbloed, W. (1) 35-43

Thomas, M.H., see Pinçon, A. (1) 195-204

Timmers, M., see Struyfs, H. (3) 813-822

Tito, P.A.L., see Miranda, L.F.J.R. (2) 609-620

Tooyama, I., see Yang, H. (3) 797-811

Torres-Jardón, R., see Calderón-Garcidueñas, L. (3) 757-770

Träber, F., see Meiberth, D. (1) 139-146

Tricoire, H., see Bouleau, S. (4) 1015-1038

Tsolaki, M., see Khondoker, M. (3) 851-864

Tung, Y.C., see Wang, X. (2) 423-435

Uemura, K., see Jingami, N. (1) 109-115

Vaingankar, J.A., see Subramaniam, M. (4) 1127-1138

Valentí-Soler, M., see Olazarán, J. (4) 1157-1173

van Duijn, C.M., see Naudé, P.J.W. (3) 733-743

Vandijck, M., see Lachno, D.R. (2) 527-542

Varga, E., G. Juhász, Z. Bozsó, B. Penke, L. Fülöp and V. Szegedi, Amyloid- $\beta_{1-42}$ Disrupts Synaptic Plasticity by Altering Glutamate Recycling at the Synapse (2) 449-456

Vargas, L., see Moreno-Grau, S. (2) 621-629

Varon, C., see Baudron, C.R. (4) 1045-1050

Vasavada, M.M., J. Wang, P.J. Eslinger, D.J. Gill, X. Sun, P. Karunanayaka and Q.X. Yang, Olfactory Cortex Degeneration in Alzheimer's Disease and Mild Cognitive Impairment (3) 947-958

Vassilaki, M., R.H. Cha, Y.E. Geda, M.M. Mielke, D.S. Knopman, R.C. Petersen and R.O. Roberts, Mortality in Mild Cognitive Impairment Varies by Subtype, Sex, and Lifestyle Factors: The Mayo Clinic Study of Aging (4) 1237-1245

Vecchio, F., F. Miraglia, G. Curcio, R. Altavilla, F. Scrascia, F. Giambattistelli, C.C. Quattrocchi, P. Bramanti, F. Vernieri and P.M. Rossini, Cortical Brain Connectivity Evaluated by Graph Theory in Dementia: A Correlation Study Between Functional and Structural Data (3) 745-756

Veerhuis, R., see Jongbloed, W. (1) 35-43

Vegas, E., see Armand-Ugón, M. (2) 407-421

Vellas, B., see Khondoker, M. (3) 851-864

Venketasubramanian, N., see Cheung, C.Y. (1) 45-56

Venneri, A., see Gardini, S. (2) 457-470

Verbeek, M.M., see Jongbloed, W. (1) 35-43

Vermeiren, Y., see Naudé, P.J.W. (3) 733-743 
Vernieri, F., see Buratti, L. (3) 883-890

Vernieri, F., see Vecchio, F. (3) 745-756

Viallet, F., see Moreau, N. (2) 581-597

Vidoni, E.D., see Watts, A. (1) 187-193

Vincent, S.R., see Yang, H. (3) 797-811

Visser, P.-J., see Jongbloed, W. (1) 35-43

Viticchi, G., see Buratti, L. (3) 883-890

von Arnim, C.A.F., see Nagel, G. (4) 1119-1126

Vorobyov, V., V. Kaptsov, R. Gordon, E. Makarova, I. Podolski and F. Sengpiel, Neuroprotective Effects of Hydrated Fullerene $\mathrm{C}_{60}$ : Cortical and Hippocampal EEG Interplay in an Amyloid-Infused Rat Model of Alzheimer's Disease (1) 217-233

Wagner, M., see Meiberth, D. (1) 139-146

Wall, A., see Leuzy, A. (4) 1077-1088

Wallin, A., see Rolstad, S. (3) 873-881

Walter, H., see Meiberth, D. (1) 139-146

Wang, F., see Qin, W. (1) 245-252

Wang, G., see Xia, Q. (2) 373-386

Wang, H., see Wang, X. (3) 781-795

Wang, H., see Xia, Q. (2) 373-386

Wang, J., see Vasavada, M.M. (3) 947-958

Wang, J., see Wang, X. (3) 781-795

Wang, L., see Sun, Y. (1) 269-282

Wang, L., see Xie, B. (3) 721-731

Wang, Q., see Qin, W. (1) 245-252

Wang, S., see Berisha, V. (3) 959-963

Wang, X., see Xie, B. (3) 721-731

Wang, X., J. Blanchard, Y.C. Tung, I. Grundke-Iqbal and K. Iqbal, Inhibition of Protein Phosphatase2A (PP2A) by $\mathrm{I}_{1}{ }^{\mathrm{PP} 2 \mathrm{~A}}$ Leads to Hyperphosphorylation of Tau, Neurodegeneration, and Cognitive Impairment in Rats (2) 423-435

Wang, X., J. Wang, Y. He, H. Li, H. Yuan, A. Evans, $\mathrm{X}$. Yu, Y. He and H. Wang, Apolipoprotein E $\varepsilon 4$ Modulates Cognitive Profiles, Hippocampal Volume, and Resting-State Functional Connectivity in Alzheimer's Disease (3) 781-795

Wang, X., O. Lopez, R.A. Sweet, J.T. Becker, S.T. DeKosky, M.M. Barmada, E. Feingold, F.Y. Demirci and M.I. Kamboh, Genetic Determinants of Survival in Patients with Alzheimer's Disease (2) 651-658

Wang, X., see Sun, Y. (1) 269-282

Wang, Y., see Wang, Y. (4) 1185-1195

Wang, Y., Y. Wang, Y. Sui, H. Yu, X. Shen, S. Chen, G. Pei, J. Zhao and J. Ding, The Combination of Aricept with a Traditional Chinese Medicine
Formula, Smart Soup, May Be a Novel Way to Treat Alzheimer's Disease (4) 1185-1195

Washimi, Y., see Ito, K. (2) 543-552

Watts, A., R.A. Honea, S.A. Billinger, K.T. Rhyner, L. Hutfles, E.D. Vidoni and J.M. Burns, A Combined Measure of Vascular Risk for White Matter Lesions (1) 187-193

Weber, S.G., see Wilkins, H.M. (1) 305-318

Weggen, S., see Richard, B.C. (4) 1223-1236

Wei, C., see Qin, W. (1) 245-252

Weigand, S.D., see Whitwell, J.L. (4) 1109-1117

Weimar, C., see Dlugaj, M. (1) 159-174

Weinmayr, G., see Nagel, G. (4) 1119-1126

Westman, E., see Khondoker, M. (3) 851-864

Whitwell, J.L., K. Kantarci, S.D. Weigand, E.S. Lundt, J.L. Gunter, J.R. Duffy, E.A. Strand, M.M. Machulda, A.J. Spychalla, D.A. Drubach, R.C. Petersen, V.J. Lowe, C.R. Jack Jr. and K.A. Josephs, Microbleeds in Atypical Presentations of Alzheimer's Disease: A Comparison to Dementia of the Alzheimer's Type (4) 1109-1117

Wilkins, H.M., S.M. Carl, S.G. Weber, S.A. Ramanujan, B.W. Festoff, D.A. Linseman and R.H. Swerdlow, Mitochondrial Lysates Induce Inflammation and Alzheimer's Disease-Relevant Changes in Microglial and Neuronal Cells (1) 305-318

Williams, M.A., D. Haughton, M. Stevenson, D. Craig, A.P. Passmore and G. Silvestri, Plasma Complement factor H in Alzheimer's Disease (2) 369-372

Willis, B.A., see Lachno, D.R. (2) 527-542

Winkler, A., see Dlugaj, M. (1) 159-174

Wirths, O., see Richard, B.C. (4) 1223-1236

Wisse, L.E.M., Y.D. Reijmer, A. ter Telgte, H.J. Kuijf, A. Leemans, P.R. Luijten, H.L. Koek, M.I. Geerlings, G.J. Biessels and on behalf of the Utrecht Vascular Cognitive Impairment (VCI) Study Group, Hippocampal Disconnection in Early Alzheimer's Disease: A 7 Tesla MRI Study (4) $1247-1256$

Wolfsgruber, S., see Meiberth, D. (1) 139-146

Wong, T.Y. see Cheung, C.Y. (1) 45-56

Woo, S.-y., see Ye, B.S. (2) 599-608

Wright, J.W. and J.W. Harding, The Brain Hepatocyte Growth Factor/c-Met Receptor System: A New Target for the Treatment of Alzheimer's Disease (4) $985-1000$

Wu, J., see Zheng, N. (2) 659-671

Wu, L., see Qin, W. (1) 245-252

Wu, Z., see Huang, Y. (1) 235-244 
Xia, Q., H. Wang, Y. Zhang, Z. Ying and G. Wang, Loss of TDP-43 Inhibits Amyotrophic Lateral Sclerosis-Linked Mutant SOD1 Aggresome Formation in an HDAC6-Dependent Manner (2) 373-386

Xie, B., H. Zhou, R. Zhang, M. Song, L. Yu, L. Wang, Z. Liu, Q. Zhang, D. Cui, X. Wang and S. Xu, Serum miR-206 and miR-132 as Potential Circulating Biomarkers for Mild Cognitive Impairment (3) 721-731

Xing, Y., see Qin, W. (1) 245-252

$\mathrm{Xu}$, S., see Sun, Y. (1) 269-282

$\mathrm{Xu}, \mathrm{S}$, see Xie, B. (3) 721-731

Yamamoto, Y., see Ito, K. (2) 543-552

Yamashita, T., see Matsuzono, K. (3) 771-780

Yanagisawa, D., see Yang, H. (3) 797-811

Yang, D.-W., see Ye, B.S. (2) 599-608

Yang, H., H. Guan, M. Yang, Z. Liu, S. Takeuchi, D. Yanagisawa, S.R. Vincent, S. Zhao and I. Tooyama, Upregulation of Mitochondrial Ferritin by Proinflammatory Cytokines: Implications for a Role in Alzheimer's Disease (3) 797-811

Yang, M., see Yang, H. (3) 797-811

Yang, Q.X., see Vasavada, M.M. (3) 947-958

Yap, M., see Subramaniam, M. (4) 1127-1138

Yap, P., see Cheung, C.Y. (1) 45-56

Ye, B.S., S.H. Choi, S.-H. Han, S.Y. Kim, D.-W. Yang, K.H. Park, H.J. Han, Y.C. Youn, B.-O. Choi, S.H. Kim, S.-y. Woo, D.L. Na and E.-J. Kim, Clinical and Neuropsychological Comparisons of EarlyOnset Versus Late-Onset Frontotemporal Dementia: A CREDOS-FTD Study (2) 599-608

Yeheskel, A., see Gozes, I. (1) 57-73

Yen, F.T., see Pinçon, A. (1) 195-204

Ying, Z., see Xia, Q. (2) 373-386

Yoo, J.-S., see Lee, D.Y. (3) 837-849

Yoshimura, A., see Bun, S. (1) 15-25

Youn, Y.C., see Ye, B.S. (2) 599-608
Yu, H., see Wang, Y. (4) 1185-1195

Yu, L., see Xie, B. (3) 721-731

Yu, X., see Wang, X. (3) 781-795

Yuan, H., see Wang, X. (3) 781-795

Yuan, P., see Zheng, N. (2) 659-671

Zaki, A.F., see Khedr, E. (1) 117-126

Železná, B., see Špolcová, A. (3) 823-835

Zemenová, J., see Špolcová, A. (3) 823-835

Zhang, C., C.-C. Kuo, S.H. Moghadam, L. Monte, K.C. Rice and R.A. Rissman, Corticotropin-Releasing Factor Receptor-1 Antagonism Reduces Oxidative Damage in an Alzheimer's Disease Transgenic Mouse Model (2) 639-650

Zhang, C., see Campbell, S.N. (4) 1175-1184

Zhang, H., J. Liu, S. Sun, E. Pchitskaya, E. Popugaeva and I. Bezprozvanny, Calcium Signaling, Excitability, and Synaptic Plasticity Defects in a Mouse Model of Alzheimer's Disease (2) 561-580

Zhang, J., see Pan, C. (3) 709-719

Zhang, Q., see Xie, B. (3) 721-731

Zhang, R., see Xie, B. (3) 721-731

Zhang, Y., see Subramaniam, M. (4) 1127-1138

Zhang, Y., see Xia, Q. (2) 373-386

Zhao, J., see Wang, Y. (4) 1185-1195

Zhao, S., see Yang, H. (3) 797-811

Zheng, N., P. Yuan, C. Li, J. Wu and J. Huang, Luteolin Reduces BACE1 Expression through NF- $\mathrm{BB}$ and Estrogen Receptor Mediated Pathways in HEK293 and SH-SY5Y Cells (2) 659-671

Zhou, A., see Qin, W. (1) 245-252

Zhou, B., see Huang, Y. (1) 235-244

Zhou, H., see Xie, B. (3) 721-731

Zhou, J., see Susanto, T.A.K. (1) 253-268

Zhu, H., see Calderón-Garcidueñas, L. (3) 757-770

Zmuda, M.D., see Koenig, A.M. (1) 175-185

Zuo, X., see Qin, W. (1) 245-252

Zweig, J.A., see Gray, N.E. (3) 933-946 